\title{
Analysis on the MRI Results of Neonatal Brain with Different Levels of Bilirubin
}

\section{LUZHONGXING ( $\square$ szdxlzx@126.com )}

Changzhou Maternal And Child Health Care Hospital https://orcid.org/0000-0002-4383-1475

SHOULING DING

Paediatric Department of Taicang First People's Hospital

\section{FEN WANG}

Paediatic Department of Taicang First People's Hospital

Haitao Lv

Soochow University Affiliated Children's Hospital

\section{Research article}

Keywords: MRI abnormality change, acute bilirubin encephalopathy, bilirubin, hyperbilirubinemia

Posted Date: March 17th, 2020

DOI: https://doi.org/10.21203/rs.3.rs-17458/v1

License: (c) (1) This work is licensed under a Creative Commons Attribution 4.0 International License. Read Full License 


\section{Abstract}

Numerous studies have focused on abnormal changes in craniocerebral MRI in neonates at severe bilirubin levels, and no abnormal changes have been reported at moderate and low levels, and the abnormal changes are related to gestational age, birth weight and bilirubin levels And other factors. Here, by analyzing the neonatal craniocerebral MRI manifestations at different levels of bilirubin, it is found that at moderate and low levels of bilirubin, the neonatal cranial MRI has abnormal changes, and the craniocerebras at severe bilirubin levels MRI abnormal changes were not statistically significant, and were not significantly related to gestational age, birth weight, and bilirubin levels. This suggests that neurotoxicity may occur at low and medium levels of bilirubin.

\section{Introduction}

The accumulation of bilirubin in the body, causing xanthochromia of skin and organs, is the most common clinical problem in the neonatal period ${ }^{[1]}$. Mainly, with the high accumulation of unconjugated bilirubin, severe patients may suffer from bilirubin encephalopathy, which will cause permanent damage to the basal ganglia, hippocampus, hypothalamus, cerebellar neurons, etc. Clinical manifestation includes lethargy, poor response, fever, apnea, convulsion and so on. Magnetic resonance imaging (MRI) has proved to be highly sensitive in bilirubin encephalopathy. MRI: high stripy signals of globus pallidus and subthalamic nucleus can be detected in early T1WI, high stripy symmetric signals of globus pallidus can be detected in later T2W| ${ }^{[2-3]}$. However, bilirubin encephalopathy caused by bilirubin only accounts for a small part of neonate hyperbilirubinemia, most of them have no clinical manifestation except jaundice. Then, whether these high accumulation of bilirubin will cause brain damage to neonates or not? Is there any difference of brain damage caused by different levels of bilirubin with the high accumulation of bilirubin? For answering these questions, we examined the brain MRI of neonates with different levels of bilirubin levels, and the MRI results are as followed:

\section{Materials And Methods}

\section{Clinical Data}

Retrospectively analyzed 103 neonates( 51 boys and 52 girls) without manifestation of bilirubin encephalopathy, who had been hospitalized in Neonatology Department of Taicang First People's Hospital from March 2013 to September 2015. Gestational age of them ranges from 31+6 weeks to 43+1 weeks(in average,38.14 \pm 2.21 weeks), birth weight from $1.55 \mathrm{~kg}$ to $5.00 \mathrm{~kg}$ (average,3.18 $\pm 0.64 \mathrm{~kg}$ ), 26 preterm neonates and 77 full-term neonates. These neonates were divided into three groups based on their levels of serum bilirubin concentration, 16 cases in mild group(TSB: $0.0 \sim 229.0 \mu \mathrm{mol} / \mathrm{L}$ ), 49 cases in moderate group (TSB: 229.0 342.0 $\mu \mathrm{mol} / \mathrm{L}$ ) and 38 cases in severe group (TSB $\geq 342.0 \mu \mathrm{mol} / \mathrm{L})$. Possible disease, such as toxic cerebral hypoxia damage, hepatolenticular degeneration, was excluded, which had similar MRI manifestation of bilirubin encephalopathy ${ }^{[2]}$. 
Methods

A Siemens Skyra 3.0T superconductivity magnetic resonance scanner and new Tim4G platform equipped with 20 units of high-density matrix head and neck joint coils. Before MRI scanning, the neonate patients were accepted a routine sedation, kept in supine position and been warm. T1WI(TR=477 ms, TE=15 ms), $\mathrm{T} 2 \mathrm{WI}(\mathrm{TR}=4060 \mathrm{~ms}, \mathrm{TE}=120 \mathrm{~ms})$, matrix $256 \times 256$, slice thickness $5 \mathrm{~mm}$, gap $0.4 \mathrm{~mm}$. Coronal scanning was done for all neonate patients. The MRI scan sequences and parameters were as follows: T1WI(TR=450 ms, TE=10 ms), T2W1(TR=4200 ms, TE=98 ms), matrix 220×256, slice thickness $5 \mathrm{~mm}$, gap $1.5 \mathrm{~mm}$.

Criteria for Diagnosis

All MRI results were jointly written and analyzed by two radiologists who did not know the clinical history of patient.

MRI: high stripy signals of globus pallidus and subthalamic nucleus detected in T1WI, or high stripy symmetric signals of globus pallidus detected in T2WI or uneven signal of basal ganglion was regards as abnormality. No above-mentioned manifestation was regarded as normality. and heterpgenous signal intensity of basilar on $\mathrm{T} 2 \mathrm{~W} 1^{[2-3]}$.

\subsubsection{Brainstem Auditory Evoked Potential(BAEP)}

BAEP examination was done for the 103 neonate patients. Passed the examination was regarded as normality, failed the examination was regarded as abnormality.

Statistical Method

Data analysis was performed by the use of SPSS 23.0 statistical software. Each measuring parameter was expressed by Mean $\pm S D()$ and t-tests (multi-group comparisons), while each counting parameter was expressed by rate and $c^{2}$ tests(comparisons among groups). $P<0.05$, the difference is statistically significant.

\section{Results}

2.1.1 Comparison of the bilirubin values of three groups

Table 1 


\begin{tabular}{llll} 
Group & Mild & Moderate & Severe \\
\hline Bilirubin value & $171.99 \pm 33.50$ & $293.98 \pm 32.09$ & $375.59 \pm 34.25$ \\
\hline $\boldsymbol{t}$ & 20.54 & 64.13 & 67.59 \\
\hline $\boldsymbol{P}$ & 0.00 & 0.00 & 0.00
\end{tabular}

The comparison of bilirubin values of the three groups of neonates (Pख0.01) indicates the difference is statistically significant.

2.1.2 Comparison of brain MRI results of the three neonates groups

Table 2

\begin{tabular}{llll} 
Group & Mild & Moderate & Severe \\
\hline Normal & 11 & 41 & 30 \\
\hline Abnormal & 5 & 8 & 8 \\
\hline $\mathbf{c}^{2}$ & & 1.579 & \\
\hline $\boldsymbol{P}$ & & 0.454 &
\end{tabular}

The comparison of brain MRI results of the three neonates groups (P凶0.05) indicates that the difference is not statistically significant.

2.2.1 Comparison of the bilirubin values between the pre-term group and the full-term group

Table 3

\begin{tabular}{|c|c|c|}
\hline Group & Pre-term & Full-term \\
\hline Bilirubin value & $289.70 \pm 85.38$ & $310.36 \pm 72.32$ \\
\hline$t$ & & \\
\hline$P$ & & \\
\hline
\end{tabular}

The comparison of the bilirubin values between pre-term group and full-term group ( $\mathrm{P} \otimes 0.05)$ indicates that the difference is not statistically significant.

2.2.2 Comparison of MRI results between pre-term group and full-term group

Table 4 


\begin{tabular}{|c|c|c|}
\hline Group & Pre-term & Full-term \\
\hline Normal & 18 & 64 \\
\hline Abnormal & 8 & 13 \\
\hline$c^{2}$ & & \\
\hline$P$ & & \\
\hline
\end{tabular}

The comparison of brain MRI results between prem-term group and full-term group (Pख0.05) indicates that the difference is not statistically significant.

2.3.1 Two groups divided based on the weight of $2500 \mathrm{~g}$

Table 5

\begin{tabular}{llll} 
Group & $\mathbf{1 2 5 0 0 \mathrm { g }}$ & & $\mathbf{2 5 0 0 \mathrm { g }}$ \\
\hline Weight & $2.04 \pm 0.21$ & & $3.39 \pm 0.46$ \\
\hline $\boldsymbol{t}$ & & -18.85 & \\
\hline $\boldsymbol{P}$ & & 0.00 & \\
\hline
\end{tabular}

The weight comparison of the two groups $(P \llbracket 0.01)$ indicates that the difference is statistically significant.

2.3.2 Comparison of MRI results of the two neonates groups divided based on the weight of $2500 \mathrm{~g}$

Table 6

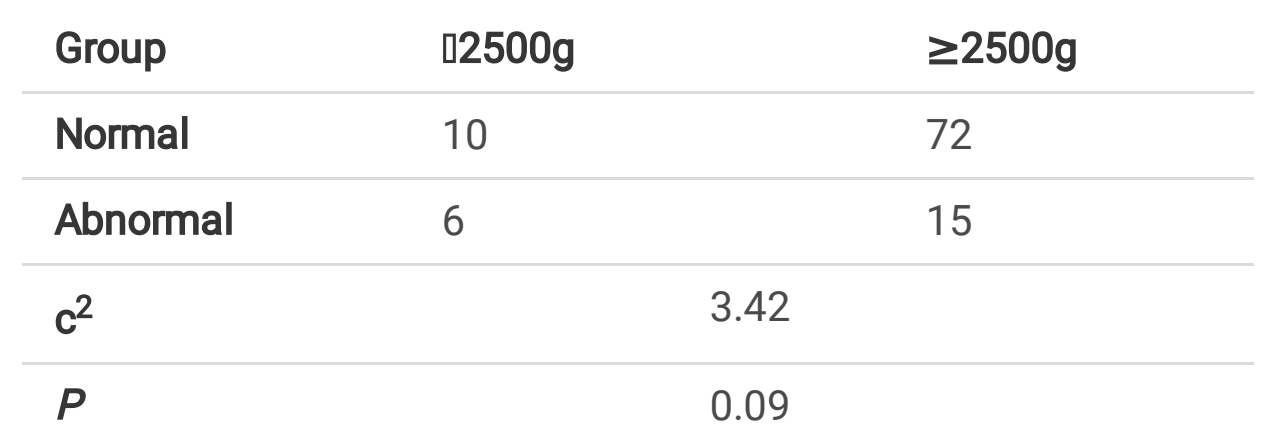

The comparison of brain MRI results of two neonates groups (Pख0.05) indicates that the difference is not statistically significant.

2.4 Comparison between normal and abnormal MRI bilirubin values

Table 7 


\begin{tabular}{|c|c|c|}
\hline Group & Normal『82】 & Abnormal|21区 \\
\hline bilirubin value & $305.55 \pm 74.54$ & $303.56 \pm 83.04$ \\
\hline$t$ & 0.11 & \\
\hline$P$ & 0.92 & \\
\hline
\end{tabular}

The comparison of bilirubin values between the two groups (Pख0.05) indicates that the difference is not statistically significant.

2.5 Comparison of abnormal MRI signal values of globus pallidus on T1WI in mild, moderate and severe groups and comparison of these abnormal MRI signal values with normal MRI signal values

Table 8

Group n $\quad \begin{aligned} & \text { Mean signal value of left globus } \\ & \text { pallidus }\end{aligned} \quad \begin{aligned} & \text { Mean signal value of right globus } \\ & \text { pallidus }\end{aligned}$

\begin{tabular}{|lccc|}
\hline Normal group ${ }^{1}$ & 82 & $892.03 \pm 132.54$ & $878.65 \pm 126.43$ \\
\hline Mild group ${ }^{2}$ & 5 & $956.85 \pm 245.87$ & $942.06 \pm 232.60$ \\
\hline $\begin{array}{l}\text { Moderate group } \\
23\end{array}$ & 8 & $1056.23 \pm 254.21$ & $1032.32 \pm 222.32$ \\
\hline
\end{tabular}

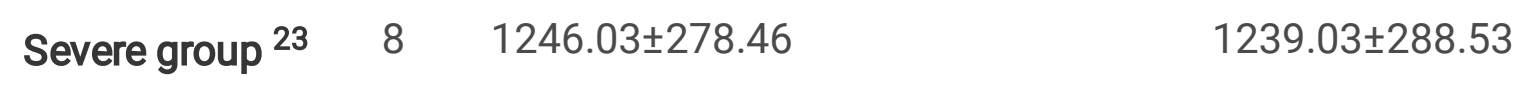

The comparison of normal group signal values with that of mild group $(p<0.05)$, with that of moderate group and with that of severe group $(p<0.01)$ indicates that the difference is statistically significant; 2 . The comparison of signal values between mild and moderate groups $(p<0.05)$ and between mild group and severe group $(p<0.01)$ indicates that the difference is statistically significant; 3 . The comparison of signal values between moderate group and severe group $(p<0.05)$ indicates that the difference is statistically significant.

2.6 Comparison of hearing screening results for neonates patients with abnormal MRI in three groups and comparison of hearing screening results between neonates patients with abnormal MRI and normal MRI.

Table 9 

Group
Normal
MRI
Abnormal MRI in
mild group $45 \square$
Abnormal MRI in
Abnormal MRI in
moderate group $[8]$
severe group $\square 8 \square$

प82П

\begin{tabular}{lllll}
\hline Normal & 67 & 3 & 4 & 2 \\
\hline Abnormal & 15 & 2 & 4 & 6 \\
\hline$\%$ & 18.29 & 40.00 & 50.00 & 75.00 \\
\hline $\begin{array}{l}\text { Reviewabnormality } \\
\text { after one month }\end{array}$ & 0 & 0 & $1 \rrbracket 12.50 \rrbracket$ & $2 \varangle 25.00 \rrbracket$ \\
\hline
\end{tabular}

\section{Discussion}

Neonatal jaundice is the most common clinical manifestation occurred in neonate period, which is mainly caused by the increase of serum bilirubin in neonates. In the prenatal period, due to the hypoxic environment, the amount of red cells is increased and the hemoglobin content in the fetal is high . After birth, due to the hyperoxic environment, the survival time of red blood cell becomes short. The fetal hemologlobin is gradually converted to adult hemoglobin producing a large amount of bilirubin, which exceeds the ability of liver to treat bilirubin. When bilirubin levels are above $139 \mu \mathrm{mol} / \mathrm{L}$, skin or organ xanthochromia may appear, that is called neonate jaundice. It has been previously thought if the concentration of bilirubin in the blood is above $342 \mu \mathrm{mol} / \mathrm{L}$, serious damage to nervous system for the neonates may occur and the acute stage manifestation of acute bilirubin encephalopathy ( $A B E)$ or chronic kernicterus appears ${ }^{[4]}$. Brain damage caused by neonatal hyperbilirubinemia(NHB) is not always reversible, may lead to cerebral palsy and hearing loss. The most common type of nerve injuries are the sequelae of severe neurological dyskinesia and hearing impairment. ${ }^{[5-8]}$. Chronic bilirubin encephalopathy or kernicterus mainly affects subcortical regions, such as globus pallidus, hypothalamus, substantia nigra, cerebellar dentate nucleus, or hippocampus. Although, the underlying mechanisms are still unknown ${ }^{[9-10]}$. Most of $A B E$ neonatal patients still have a chance to be recovered by timely treatment of reducing serum bilirubin levels, while only a few suffer from sequelae, like kernicterus.

The feature of MRI manifestation to bilirubin encephalopathy is the high signal of globus pallidus in the acute stage of T1-weighted imaging (T1WI). With the development of the disease, it has changed from the high signal in acute stage of $\mathrm{T} 1 \mathrm{WI}$ to the high symmetric signal of bilateral globus pallidus and subthalamic nucleus in chronic stage and FLAIR sequence signal ${ }^{[11-13]}$. Since in the neonatal period the basal ganglia nerve cells have strong physiological and biochemical metabolism and the oxygen consumption is increasing, especially, the middle and later part of the globus pallidus is the most sensitive ${ }^{[14]}$. This leads to the selectively deposition of serum bilirubin in the globus pallidus, the middle and later part of the globus pallidus is more sensitive ${ }^{[15-17]}$. Besides, it will cause the damage to neurons and glial cells, the apoptosis of neurons and the change of glial cells' mitochondria function ${ }^{[18-19]}$. Basal ganglia pallidus injury caused by hyperbilirubinemia can be efficiently detected by MRI. The high and 
symmetric signal of bilateral T1WI pallidal is an important imaging feature of neonatal $A B E$, while the change from high signal of T1WI to high signal of T2-weighted imaging (T2W1) is the imaging feature of nuclear jaundice on MRI, indicating neuronal cell necrosis and poor prognosis. Presently, the mechanism of MRI signal change still remains unclear. This may be related to the reduction of the T1 value, which is caused by the deposition of bilirubin in glial cells and the destruction of bilirubin to nerve cell membrane $[20]$

Most of the MRI results mentioned above were obtained from brain MRI findings of the severe or profound NHB patients with bilirubin encephalopathy or non-bilirubin encephalopathy. So far, there is no report about whether the MRI results of mild and moderate NHB patients without bilirubin encephalopathy manifestation are abnormal. Therefore, this study retrospectively analyzes the bilirubin level and MRI results of 103 patients with jaundice in order to understand whether there is abnormal brain MRI at different bilirubin levels, as well as whether there is statistical difference of abnormal brain MRI results in bilirubin levels, gestational age, and birth weight.

It is reported in literature that some mild levels of bilirubin could cause temporary or permanent neurological sequelae under the condition that a certain level of bilirubin is considered as being safe by people ${ }^{[21]}$. Based on total serum bilirubin concentration(TSB), the 103 patients in our research were divided into three groups, including 16 cases in the mild group (TSB: $0.0-229.0 \mu \mathrm{mol} / \mathrm{L}$ ), 49 cases in the moderate group (TSB: $229.0-342.0 \mu \mathrm{mol} / \mathrm{L}$ ) and 38 cases in the severe group ( TSB $\geq 342.0 \mu \mathrm{mol} / \mathrm{L}$ ). There were 21 cases with abnormal MRI results, which consisted of 5 cases in mild group, 8 cases in moderate group and 8 cases in severe group. The comparison of TSB among the three groups $P \otimes 0.01$ indicated that the difference was statistically significant, whereas, the comparison of abnormal brain MRI results among the three groups $\mathrm{P} \otimes 0.05$ indicated that the difference was not statistically significant. This pointed out that bilirubin brain nerve damage may occur in patients without the manifestation of $A B E$ even in low level of bilirubin, and the rate of MRI abnormality is not rising with the rising of bilirubin level. The study findings are not consistent with the reports of Changjun Ren et al. that the higher the total bilirubin level is, the higher the proportion of abnormal MRI is ${ }^{[22]}$. We think that low number of cases in the mild group may also be an important factor. Under Taoka et al.'s follow-up of the observed subjects for 2 years, it was found that the infants had been developing normally whether the high symmetric T1WI signal was demonstrated in GP and STN regions or not. In accordance with reports in the literature, it may be related to the development of gray matter ball in GP and STN after the birth of neonates ${ }^{[23-26]}$. Besides, lots of literatures have reported that this manifestation is one of the brain MRI findings of nuclear jaundice ${ }^{[27-30]}$. Along with the extensive clinical application of MRI, the manifestation is becoming more and more common in neonates, and normal neonates. Thus, some scholars recently have raised different opinions ${ }^{[22-24]}$. For example, Hrris et al. ${ }^{[31]}$ reported that high symmetric signal in the globus pallidus region of four 5-21 day neonates with acute kernicterus has disappeared during the follow-up. This manifestation was thought transient, had no correlation with the prognosis of the patients. This manifestation also appeared in the cases of neonatal hypoxic ischemic encephalopathy, hypoglycemia and other cases ${ }^{\text {[32-33]. }}$ 
In this study, the neonate patients, who has high signal of globus pallidus in T1WI or T2W1, caused by the diseases of neonatal hypoxic ischemic encephalopathy, hypoglicrmia, hepatolenticular degeneration and other diseases, have been excluded. In this study, 12 cases of the 21 patients with MRI abnormalities were followed up for one month, and 9 cases of the 21 patients were followed up for 3 months. 6 cases of patients had normal brain MRI results in the 1 month later reexamination. 3 cases of patients had not been reexamined of the MRI, one of the 3 cases had neurological abnormalities 2 months later, who was delivered with gestational age 40 weeks, birth weight $3.31 \mathrm{~kg}$, clear amniotic fluid, the Apgar score 8 at 1 min and 9 in 5 mins, maximum bilirubin value $327.3 \mu \mathrm{mol} / \mathrm{L}$, and uneven signal of the brain MRI in bilateral basal ganglia region. However, one case of this study showed no brain MRI abnormalities with maximum bilirubin value $502.3 \mu \mathrm{mol} / \mathrm{L}$, birth weight $3.05 \mathrm{~kg}$, gestational age $36+3$ weeks, clear amniotic fluid and the Apgar score 9 at $1 \mathrm{~min}, 9$ in 5 mins. In addition, another case in the study may have ABE with gestational age 40+4 weeks, birth weight $3.5 \mathrm{~kg}$, amniotic fluid in III meconium-stained during birth, the Apgar score 9 at $1 \mathrm{~min}, 9$ in 5 mins, the maximum bilirubin value $130.4 \mu \mathrm{mol} / \mathrm{L}$, and high symmetric T1WI signal of bilateral basal ganglia indicated by his brain MRI. The high symmetric signal of bilateral globus pallidus is not unique to the neonatal $A B E$, it can also be seen in some neonatal patients with hypoxic ischemic encephalopathy ${ }^{[34-35]}$, or even in normal neonates ${ }^{[36]}$. But the MRI manifestations of hypoxic ischemic encephalopathy (HIE) involved more extensive scope, which was characterized by internal capsule, the putamen and the thalamu, and accompanied by cortical and subcortical, deep leukoplakia plaque abnormal signals, diffuse brain edema, intracranial hemorrhage, and so on. These accompanied manifestations were scarcely appeared in bilirubin encephalopathy ${ }^{[37]}$. Without the medical history, signs of hypoxic ischemic encephalopathy and the above MRI manifestations, the symmetric T1WI high signal was demonstrated in bilateral basal ganglia of the child brain MRI

Blood brain barrier injury, might be caused by anoxia or other factors in antepartum and intrapartum, increased the permeability of blood brain barrier, resulting in the increasing of free bilirubin to enter into brain tissue through the injured blood-brain barrier and to deposit in the basal nerve nucleus, cerebral ganglia, subthalamic nucleus, parietal nucleus, ventricular nucleus, caudate nucleus, cerebellum, oblongata, cerebral cortex and spinal cord. Accordingly, the utilization of oxygen in brain tissue was inhibited, leading to brain damage ${ }^{[38-39]}$. It is also believed that bilirubin deposition cause the influx of neuron cells $\mathrm{Ca}^{2+}$ and stimulate the increase of proteolytic enzyme activity, leading to neuronal apoptosis $^{[36]}$.

It has been reported in many studies that the occurrence of neonatal BE is related to the factors of gestational age, birth weight, bilirubin binding state and bilirubin level, etc. ${ }^{[18]}$ In this study, 103 patients were divided into preterm group (26 cases) and full-term group (77 cases), observing the comparison of bilirubin values $(289.70 \pm 85.38 v s 310.36 \pm 72.32, P=0.232)$ of the two groups and the MRI abnormal result $(P=0.16)$ of the two groups was $P \otimes 0.05$, the difference was not statistically significant, which indicated that there may be no difference in the toxicity of bilirubin to central nervous system between pre-term group and full-term group without BE clinical manifestation. Moreover, it was not case that premature infants with younger gestational age were more susceptible to bilirubin toxicity. The analyses of this 
result may be as follows: (1) Premature delivery patients, who are usually admitted to be in hospital after birth, are treated for jaundice in time during hospitalization. While, full-term infants are admitted to be in hospital only when their bilirubin value reaches a higher level. On the one hand, high level of bilirubin could easily cause nerve damage by through the blood brain barrier; on the other hand, the longer the high level of bilirubin remains in the body, the more neurotoxicity of bilirubin is. (2) The statistical analysis in this study may be biased, on account of the fewer cases of pre-term delivery group, especially lack of preterm patients less than 31 weeks of gestational age due to obstetric factors. Meanwhile, we also observed whether there was statistical difference between birth weight and brain MRI abnormality of the jaundice patients. Based on birth weight, 103 patients were divided into two groups, one group birth weight $<2500 \mathrm{~g}$ ( 16 cases, including 6 abnormal cases) and the other group birth weight $\geq 2500 \mathrm{~g}$ (87 cases, including 15 abnormal cases). The comparison of brain MRI abnormality of the two groups was $P=0.09, P>0.05$, the difference was not statistically significant .

In addition, the comparison of bilirubin value between abnormal MRI group and normal MRI group was (303.56 \pm 83.04 vs $305.55 \pm 74.54, P=0.92, P>0.05)$, the difference was not statistically significant, which indicated that there was no obvious difference in bilirubin level between abnormal MRI group and normal MRI group of jaundice patients without ABE.

At the same time, we measured the T1WI signal values of the patients with abnormal brain MRI and the signal values of the patients with normal brain MRI in the three groups, and performed statistical comparison, which showed that the T1WI signal values of the patients with abnormal MRI were all higher than those of the patients with normal MRI $(P<0.05)$, and the T1WI signal values of the patients with MRI abnormalities in the three groups also had statistically difference $(P<0.05)$. With the increase of serum bilirubin level, the T1WI signal value of the patients with MRI abnormalities also increased, which was consistent with the report of Yan Ruifang et al ${ }^{[40]}$ that there was a linear correlation between the mean signal value of globus pallidus and serum total bilirubin levels in the lesion group. This indicated that with the rising of bilirubin level, the more bilirubin deposited on neuron such as globus pallidus, the more serious damage would be made to nerve tissue such as neuron.

We performed hearing tests for the 103 patients with BAEP ${ }^{[41]}$ devices, it was found that 15 cases of 82 patients with normal MRI results were abnormal, accounting for $18.29 \%$. In the mild group 2 cases of 5 patients with abnormal MRI were abnormal, accounting for $40.00 \%$. In the moderate group 4 cases of 8 patients with abnormal MRI were abnormal, accounting for $50.00 \%$. In the severe group 6 cases of 8 patients with abnormal MRI were abnormal, accounting for $75.00 \%$. After one month followed-up, the reexamination found that the patients with hearing abnormalities in the MRI normal group and the mild group were all returned to normal level, and 1 case in the moderate group and 2 cases in the severe group were remained abnormal. This result indicated that, on the one hand, the BAEP abnormal rate of the patients with abnormal MRI was higher than that of the patients with normal MRI, and the BAEP abnormal rate of the patients with abnormal MRI increased with the increase of bilirubin level. It also shows that with the increase of bilirubin, the hearing damage is more obvious ${ }^{[42]}$. On the other hand, it also proved the application value of MRI in neonatal jaundice patients, which could timely detect the 
abnormalities of central nervous system of the patients with jaundice, and provide imaging evidence for early diagnosis and early intervention.

In conclusion, in the presence of certain factors, central nervous system damage may also occur at low level of bilirubin and be appeared abnormality on MRI. Meanwhile, MRI can also be used to provide early imaging signs for the judgment of central nervous system damage to NHB neonatal patients without clinical manifestations of $A B E$, and offer clues for early treatment and early intervention so as to prevent the occurrence of severe brain tissue damage and over-treatment of the patient who only has hyperbilirubinemia ${ }^{[43]}$.

\section{Limitations Of The Present Study}

Although the findings of this study indicate that in babies with jaundice, the brain MRI may show abnormal changes at mild and moderate levels of bilirubin, and there is no statistically significant difference between brain MRI abnormalities in the severe group, Our results have been affected by a number of issues. First, we failed to measure the free bilirubin levels, internal environmental conditions such as serum albumin levels, $\mathrm{pH}$ values, etc. of the jaundice newborns participating in the study at that time, so it cannot be said with certainty that the baby's brain was exposed to biliary red crossing the blood-brain barrier Prime type. Secondly, the sample size in this study is relatively small, especially in the mild group and the sample size is less than $2500 \mathrm{~g}$. Finally, the craniocerebral pathology test of newborn animal Jaundice model is lacking to validate our findings. This will be an issue that we need to address in this type of research in the future.

\section{Abbreviations}

ABE : acute bilirubin encephalopathy

TSB : total serum bilirubin

MRI: Magnetic resonance imaging

NHB : neonatal hyperbilirubinemia

ABE : acute bilirubin encephalopathy

T1WI :T1-weighted imaging

T2WI :T2-weighted imaging

\section{Declarations}

These authors have contributed equally to this work. 
Compliance with Ethical Statements: The study was approved by the Ethics Committee of the First People's Hospital of Taicang City.

Conflict of interest statement: The authors claim no conflict of interest.

Funding: There is no funding source.

Ethical approval: This article does not contain any studies with human participants or animals performed by any of the authors.

Informed consent: All parents of participants provided written informed consent for participation in this study.

\section{References}

1. Yonghao Gui, Xindong Xue, Lizhong Du, et al. Pediatrics [M], Version 3, Beijing: People's Medical Publishing House, 2015: 130-134.

2. Binbin Ye, Guoguang Fan, Miao Fan, et al. Pediatric Imaging Diagnosis and Clinic [M], Version 1 , People's Military Surgeon Press, 2009: 491-493.

3. Congle Zhou, Zezhong Tang, Xilin Hou, et al. Neonatal Neurology [M], People's Medical Publishing House, 2012: 3423-426.

4. Kaifan Zhuang, The Value of Cranial MR in the Diagnosis of Neonatal Hyperbilirubin Encephalopathy [J]. Modern Diagnosis and Treatment, 2015 (6): 1332-1334.

5. Shapiro SM. Chronic Bilirubin Encephalopathy: Diagnosis and Outcome[J]. Semin Fetal Neonatal Med 2010; 15: 157-1

6. Shapiro SM. Kernicterus. In: Stevenson DK, Maisels MJ,Watchko JF, editors. Care of the Jaundiced Neonate [J].New York:McGraw-Hill; 2012. p. 229-2

7. Shapiro SM, Popelka GR. Auditory Impairment in Infants at Risk for Bilirubin-induced Neurologic Dysfunction[J]. Semin Perinatol 2011;35: 162-1

8. Campistol J, Galvez H, Cazorla AG, Ma'laga I, Iriondo M, Cusı ' V. Neurological Dysfunction Induced by Bilirrubin[J]. Neurologia, 2012; 27: 202-211.

9. Watchko JF, Painter MJ, Panigrahy A. Are the Neuromotor Disabilities of Bilirubin-induced Neurologic Dysfunction Disorders Related to the Cerebellum and Its Connections? [J] Semin Fetal Neonatal Med 2015, 20: 47-51.

10. Wisnowski JL, Panigrahy A, Painter MJ, Watchko JF. Magnetic Resonance Imaging of Bilirubin Encephalopathy: Current Limitations and Future Promise[J]. Semin Perinatol 2014;38:422-428.

11. Parashari UC, Singh R, Yadav R, Aga P. Changes in the Globus Pallidus in Chronic Kernicterus [J]. J Pediatr Neurosci 2009;4: 117-11

12. Magalha es MOGM, Brası 'lia DF. Kernicterus, Chronic Bilirubin Encephalophy: Imaging Features and Clinical Correlation[J]. ECR2016/ C-2261. 
13. Ribeiro BN, Lima GA, Ventura N, Gasparetto EL, Marchiori E. Chronic Kernicterus: Magnetic Resonance Imaging Findings[J]. Radio Bras 2016;49:407e8.

14. Yokochi K囚Magnetic Resonance Imaging in Children Kemictems[J].Act Paediatr,1995,84(8)ष937939.

15. Cece H, Abuhandan M, Cakmak A, Yildiz S, Calik M, Karakas E,et al. Diffusion-weighted Imaging of Patients with Neonatal Bilirubin Encephalopathy[J].Jpn J Radiol 2013;31:179-185.

16. Yokochi K. Magnetic Resonance Imaging in Children with Kernicterus[J].Acta Paediatr 1995;84:93793

17. Gkoltsiou K, Tzoufi M, Counsell S, Rutherford M, Cowan F. Serial Brain MRI and Ultrasound Findings: Relation to Gestational Age, Bilirubin Level, Neonatal Neurologic Status and Neurodevelopmental Outcome in Infants at Risk of Kernicterus[J].Early Hum Dev 2008;84:829-838

18. Junfeng Lv, Bingyan Yang, Weiqiong Wang, et al. Risk Factors of Acute Bilirubin Encephalopathy in Neonates With Severe Hyperbilirubinemia [J]. Chinese Journal of Neonatology,2014,29 (4):242区

19. YL Wang, Huiheng Yan, Yong Zhang, et al. Analysis of Internal and External High Risk Factors in Children with Bilirubin Encephalopathy [J]. Chinese Journal of Neonatology, 2015,30(2):90-91.

20. Ruiqi Li, Jianjie Wang, Xuxia Ge, et al. Application of 3.0T Magnetic Resonance Imaging in Early Diagnosis of Neonatal Bilirubin Encephalopathy[J]. China Medical Herald, 2016, 13(13):117-121.

21. Miyaoka T, Yasukawa R, Mizuno S, et al. Proton magnetic resonance spectroscopy (1H-MRS) of hippocampus, basal ganglia, and vermis of cerebellum in schizophrenia associated with idiopathic unconjugated hyperbilirubinemia(Gibert's syndrome)[J]. J. Psychiatr Res, 2005,39:29.)

22. Changjun Ren, Ling Hao, Sisi Cheng, et al. To Explore the Correlation Between Total Bilirubin / Albumin Ratio and Abnormal MRI Imaging in Neonates with Severe Hyperbilirubinemia[J]. Chinese Journal of Child Health Care, 2016, 24(5):499-501.

23. Taoka T, Aida N, Ochi T, et al. Transient Hyperinteusity in the Subthalamic Nucleus and Glohus Pallidus of Newborns on T1-weighted images [J]. AJNR Am J Neuroradiol, 2011, 32(6):1130-1137》

24. Barkovich AJ『KiOS BO凶Jackson DE Jr囚et al『Normal Maturation of the Neonatal and Infant Brain $\square M R$ Imaging at 1.5T[J]. Radiology, 1988, 166(1 Pt 1):173-180.

25. Counsell SJ,Maalouf EF, Fletcher AM, et al. MR Imaging Assessment of Myelination in the Very Preterm Brain [J]. AJNR Am J Neuroradiol, 2002,23(5):872-88 1.

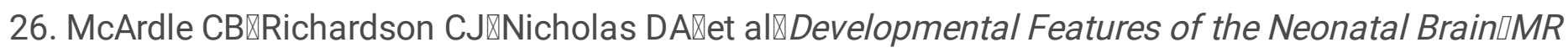
Imaging. Part I $\square$ Gray-white Matter Differentiation and Myelination [J]. Radiology, 1987, 162(1 Pt 1):223-229ه

27. Penn AA, Enzmann DR, Hahn IS, et al. Kemictems in a Full-term Infant [J]. Pediatrics, 1994, 93(6 Pt 1):1003-1006ه

28. Govaert P, Lequin M, Swarte R, et al. Changes in Globus Pallidus with (pre)term Kemicterns [J].Pediatrics, 2003, 112(6 Pt 1)I1256 -1263区 
29. Coskun A, Yikilmaz A, Kumandos, et al. Hyperintense Globus Pallidus on T1-weighted MR Imaging in Acute Kernicterus: Is It Common or Rare? [J]. Eur Radial, 2005, 15(6):1263-1276ه

30. Jian Mao, Jianhua Fu, Liying Chen, et al. MRI Character and Clinic Value of the Globus Pallidus in Neonates with Severe Hyperbilirubinemia [J]. Chinese Journal of Pediatrics, 2007, 45(1): 24-29ه

31. Harris MC囚Bembaum JC『Polin JR囚et al. Developmental Followup of Breastfed term and Near-term Infants with Marked Hyperbilirubinemia [J]. Pediatrics, 2001, 107(5):1075-1080

32. De Vries LS, Groenendaal F. Patterns of Neonatal Hypoxic Ischaemic Brain Injury [J]. Neumradiology, 2010, 52(6):555-566区

33. Figatowska M, Mierzewska H, Jurkiewicz E. Basal Ganglia Lesions in Children and Adults [J]. Eur J Radiol, 2013, 82(5):837-849

34. Cece $\mathrm{H}$, Abuhandan M, Cakmak A, et al. Diffusion-Weighted Imaging of Patients with Neonatal Bilinlbin Encephalopathy[J].Jpn J Radiol, 2013, 31(3):179-185.

35. Hui He, Yumin Lu, Analysis of Newborn High Nilirubin Encephalopathy Head MR Changes and Its Clinical Significance [J]. Chinese Modern Medicine, 2014, 2I(11): 109-111.

36. Barkovich J『MR of the neonatal neonatal bmin: Assessment of Deep stIllctures[J]『AJNR囚1998囚19(8): 1397-1403.

37. Weihua Liao, Xiaoyi Wang, Wulin Wu, et al. Differentiation of Hypoxic-ischemic Eneephalopathy and Acute Bilirubin Encephalopathy with Magnetic Resonance Imaging in Neonates [J] .Chinese Journal of Contemporary Pediatrics, 2009, 11(13):181-184.

38. Liping Liu. Effect of Different Serum Total Bile Acid Levels on Perinatal Outcome of Early Intrahepatic Cholestasis of Pregnancy [J]. Journal of Chinese Doctors,2014,42(10): 39-42.

39. Lingling Xiao, Xuefeng Zhang, Xinyu Wang, et al. Site Selection of Percutaneous Bilirubin Determination in Term Neonatal Jaundice [J]. Chinese Journal of Medicine, 2014, 49(8): 93-95.

40. Ruifang Yan, Mingdong Han, Shouying Wang, et al. Diagnostic Value of MRI and MRS in Neonatal Hyperbilirubinemia [J]. Journal of Clinical Radiology, 2014, 33(11): 1743-1747.

41. Kimitaka Kaga , Eiji Kitazumi , Kazuo Kodama. Sensorineural hearing loss in patients with cerebral palsy after asphyxia and hyperbilirubinemia [J]. International Journal of Pediatric Otorhinolaryngology (2005) 69, 1211-1217

42. Kaga, M. Suzuki, S. Koyama, Neonatal asphyxia and hyperbilirubinemia[J].JOHNS 16 (2000) 16951699 .

43. Xiaojiao Wang, Min Jiang, Yajuan Wang. Advances in Early Monitoring of Neonatal Bilirubin Encephalopathy [J]. Chinese Journal of Neonatology, 2014,29(6): 427-429.

\section{Figures}



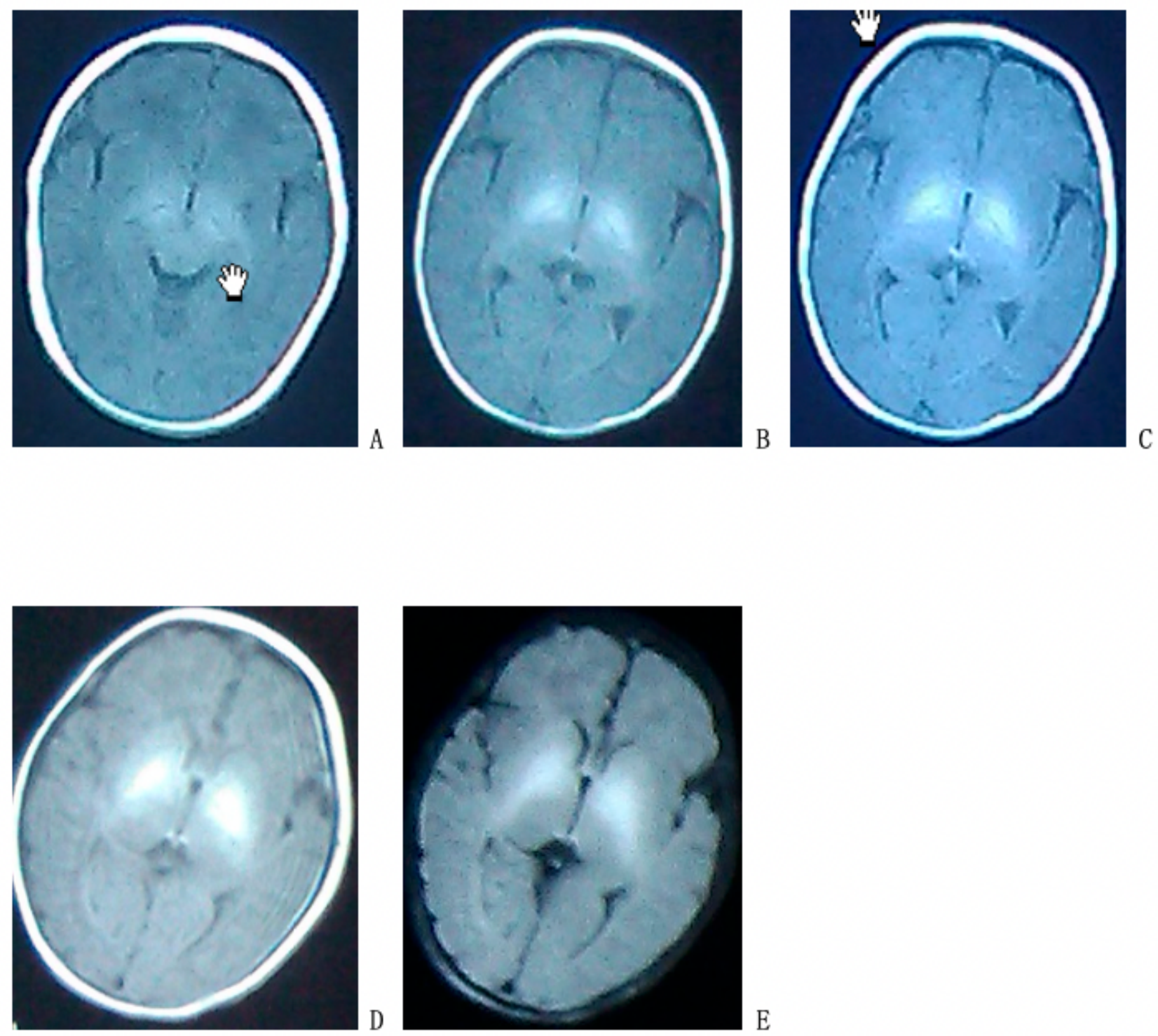

Figure 1

MRI pictures. A normal B mild C moderate D severe E T2WI manifestation 